\title{
Research on the Theme of "Chubby Baby" in Dongfengtai New Year Paintings
}

\author{
Jingyu Wang ${ }^{1, *}$
}

\author{
${ }^{1}$ Wuhan Textile University, Wuhan, Hubei, China \\ *Corresponding author. Email:819708393@qq.com
}

\begin{abstract}
At the junction of eastern Tianjin and Hebei, there is a "Sister New Year painting" — Dongfengtai New Year painting, which belongs to the same branch as Yangliuqing New Year paintings. Dongfengtai is located at the junction of Tianjin City and Tangshan City, Hebei Province. Dongfengtai New Year painting is one of the most representative folk art in this place. It has extremely high research value. Unfortunately, due to the influence of the war and the Tangshan Earthquake, it gradually disappeared. At present, there are few researches on Dongfengtai New Year paintings in the literature. Most of them only analyze and summarize the historical context and inheritance status of Dongfengtai New Year paintings. It is almost difficult to find an analysis of the characteristics of their creative styles. This article focuses on the image of "chubby baby" in Dongfengtai New Year's paintings, and compares it with Tianjin Yangliuqing's New Year paintings. It analyzes and studies the characters in Dongfengtai New Year paintings, their body characteristics, and auxiliary patterns, and then continues and promotes the cultural connotation and artistic charm of the traditional Dongfengtai New Year paintings.
\end{abstract}

Keywords: Dongfengtai, New Year paintings, Chubby baby.

\section{INTRODUCTION}

Dongfengtai is located at the junction of Tianjin City and Tangshan City, Hebei. Folk arts here are very rich. Pottery, New Year paintings, papercutting, straw weaving, dough sculptures, etc. are all popular folk arts here. Dongfengtai New Year painting is one of the most representative folk art here, and it has extremely high research value. With the changes of the times, the powerful Yangliuqing youth paintings have had a huge impact on the local New Year paintings in Dongfengtai. The style boundary between Dongfengtai New Year paintings and Yangliuqing New Year paintings has gradually become blurred, causing people to misunderstand that Dongfengtai New Year paintings are a branch of Yangliuqing New Year paintings for a long time, and they are classified into the same art system, ignoring the independence of Dongfengtai New Year paintings.

After field visits, experts interview and corresponding video data collection, and inquirie on Yangliuqing New Year painting literature. This article focuses on the image of "chubby baby" in Dongfengtai New Year's paintings, and compares it with Tianjin Yangliuqing's New Year paintings. It carries out a detailed comparative analysis of the characters in Dongfengtai New Year paintings, such as facial portrayal, posture characteristics, and auxiliary patterns. The first chapter of this article describes the historical context of Dongfengtai New Year paintings and the changes of the times; the second chapter is the positioning of Dongfengtai's style, which is a concrete sensory excavation of Dongfengtai New Year paintings from the perspective of contemporary people; the third chapter is the discussion on the subject of chubby babies in Dongfengtai New Year's paintings, mainly for analysis and comparison with Yangliuqing's New Year paintings. 


\section{RETROSPECT OF DONGFENGTAI NEW YEAR PAINTINGS RESEARCH AND STYLE CHANGES}

\subsection{History and Research Status of Dongfengtai New Year paintings}

Dongfengtai woodcut New Year paintings began in the late Ming Dynasty and flourished in the mid-Qing Dynasty. There were scenes of "every family can dye, and they are good at paintings". In the Yangliuqing Chaomidian, it is known as "Fengtai Gang" and "Hedong Goods". [2]

Since the middle of the Qing Dynasty, Dongfengtai New Year paintings have been influenced by Yangliuqing's New Year paintings, and the degree has gradually increased. During the Japanese invasion of China, Dongfengtai New Year paintings really turned from prosperity to decline. It was little known after the Tangshan earthquake in the last century.

In recent years, people's awareness of the protection of local culture has gradually increased, and there are still some old local artists who are still sticking to and embarking on digging. Some scholars also said: "Most of the New Year paintings we find in Dongfengtai today clearly have the style of Yangliuqing. Even if some paintings can see some of the regional atmosphere of Dongfengtai, most of the paintings are like Yangliuqing." 2 [1]

\subsection{The Evolution of the Style of Dongfengtai New Year Paintings}

Early Dongfengtai New Year paintings are very different from Yangliuqing's New Year paintings in terms of subject matter and artistic style. Dongfengtai New Year paintings are more rustic, simple and heavy in the north. If Dongfengtai New Year's paintings are "farmers", then Yangliuqing's paintings are "commercial public works". Old people often say, "In terms of quality, Yangliuqing is the best, and Dongfengtai is the first in terms of quantity." ${ }^{3}[3]$

1. Feng Jicai, The Lost Dongfengtai New Year Paintings [N]. China Culture Daily, 2011-09-20 (006).

2. Xing Ye, The Newly Discovered Dongfengtai Yuelongzhuang New Year paintings [J]. New Year Painting Research, 2011(0):128-130.

3. A Ying, Collected Works of A Ying (Vol. 8) [M].
In the mid-term, with the continuous growth of Dongfengtai New Year's paintings, Yangliuqing's paintings had a great influence on Dongfengtai $\operatorname{artists}^{4}[4]$. Although traditional Chinese folk art is separated from the court art, it will be influenced from the top down, from the court to the local to the folk. In the later period, people gradually ignored the independence of Dongfengtai's annualization.

\section{DONGFENGTAI NEW YEAR PAINTINGS STYLE ORIENTATION}

\subsection{Characteristics of the Characters}

In the early days of Dongfengtai New Year paintings, most of them were priests entrusting the good wishes of the folks, and they were mainly the kitchen king, the god of wealth, and the door god. Only in the middle and late stages did some other New Year painting themes other than the priesthood gradually appear, such as chubby babies, maids, and operas.

Dongfengtai New Year paintings have a certain degree of standardization in the performance of the priesthood, and people cannot make changes at will. Chubby babies and maids are not as restrained as priests, but are more free, vivid and realistic. There are many hand-painting steps, such as powdering the face, opening eyebrows, and hooking up watermarks. Some priests also need to be put golden tinfoil on the face of the statue, which people call the golden three cuts.

The opening of eyebrows in the depiction of Dongfengtai figures is quite distinctive, and it can be clearly distinguished from other New Year paintings, especially in some places where there are no eyebrows or faces. The character eye artists use the colorful colors of ink to make their eyes more realistic, and the sense of reality is very important in the eyes of Dongfengtai people. It is also one of the characteristics to paint what they see without making redundant repetitions. Fengtai people also attach great importance to the "finishing touch" opening eyebrows: they use thick ink to paint on the heaviest eyelids and eyelids; then, they use medium-density ink to draw the eyebrows, and finally use light ink to modify the lower eyelids of the eyes. The eyes drawn in this way are called

4. Niu Jiazhi, Relying on Universities to Explore the New Development Path of Beijing-Tianjin-Hebei Woodcut New Year paintings $[\mathrm{J}]$. Inner Mongolia Science Technology \& Economy, 2018(20): 121-123. 
"living eyes", which are mostly used for the eyebrows of babies and maids, as shown in the

"Figure 1".

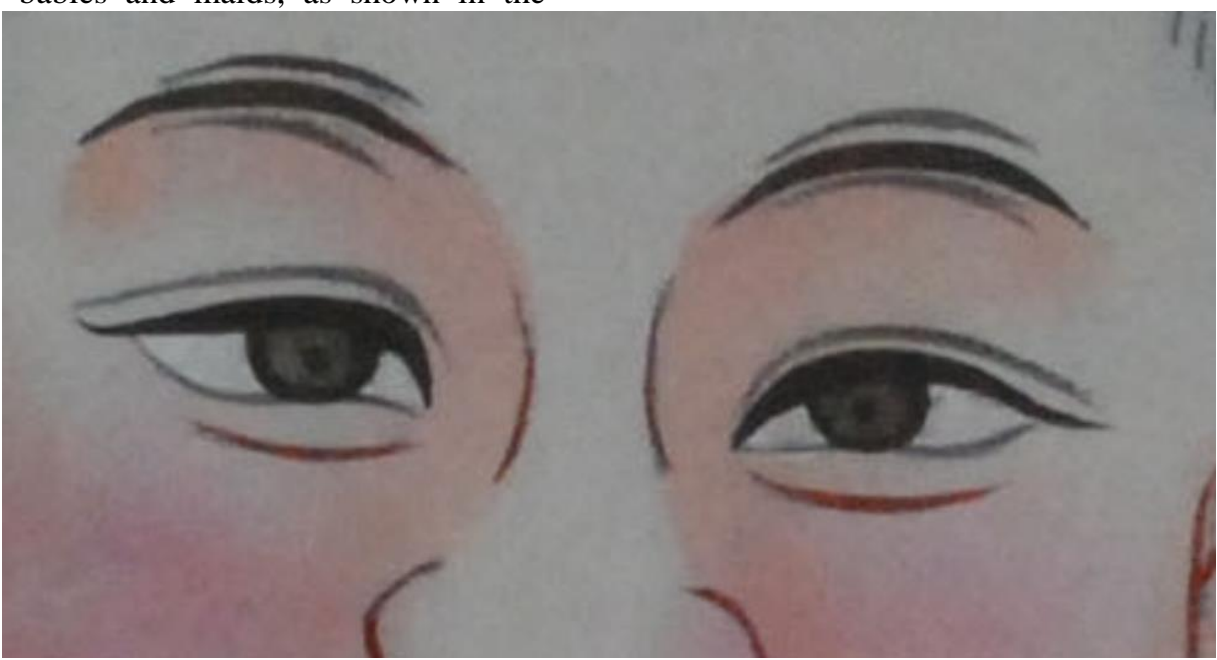

Figure 1 Part of "Lian nian you yu".

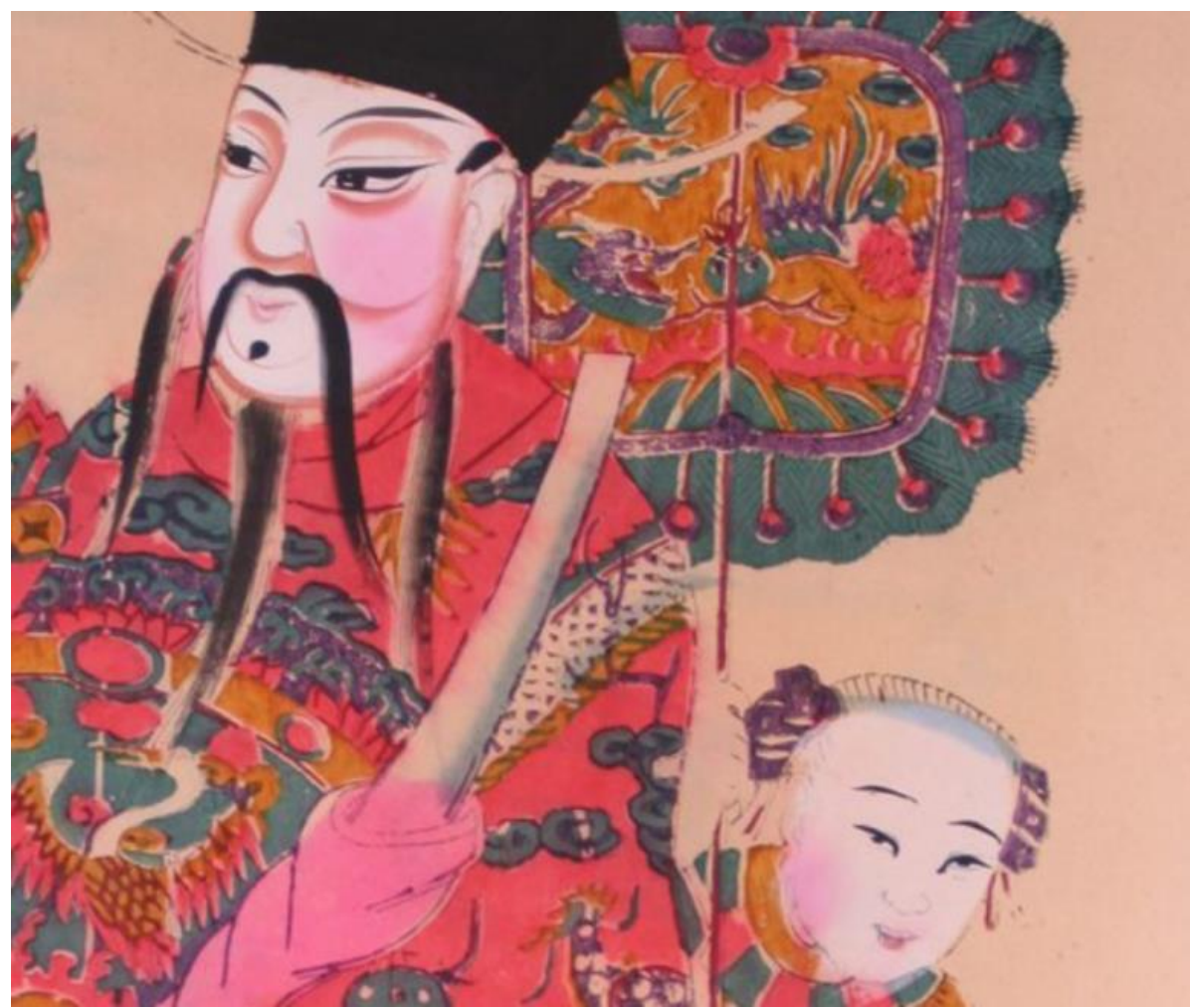

Figure 2 Part of "the God of Wealth".

The eyes drawn in this way are closer to the eyes of real characters, and they are very realistic. However, among the themes of the New Year paintings of the priesthood, the waiters and other small characters around the grandfather and grandma of the kitchen king are mostly dead eyes, as shown in "Figure 2". In addition, the painting method of "dead eye" of the priesthood is relatively simple, with long upper eyelids, and short lower eyelid, and a little point in the middle of the unsealed mouth is a "dead eye". The dead eye is not as rich as the living eye. It simply outlines the shape of the eye with ink, and the expression is relatively weak. Because it resembles the character "工" in Chinese characters, it is also called the "工"shaped eyes. In the performance of the eyes of the characters, it can be roughly divided into the maid doll category as "live-eye", and then subdivided 
into the maid's "(phoneix eye)" and the baby's "Yuanbao eye", and the paintings of clergy category are mostly in "工-shaped eyes".

\subsection{Color Usage and Meaning}

In the use of colors, the color contrast and saturation of the Dongfengtai New Year paintings are very high, and no toning is performed during the painting process. It fits the rustic artistic atmosphere of rural culture and the aesthetic orientation of the people. The bright red and green colors not only give people a very strong color feeling, but most of the red and warm colors are extraordinarily festive.

The color materials of Dongfengtai New Year paintings are all made of natural mineral pigments. In the use of colors, blue is mostly light blue, and green is light green. It does not need to be toned, only the primary colors are used, and the coloring is heavy, and the contrast is quite strong. Dongfengtai New Year paintings do not pay attention to the harmony of color blocks as in ordinary paintings. Not only do not need to pay attention to the fusion of those colors, but also the limited colors are staggered as much as possible to create the colorful effect. Dongfengtai New Year paintings are much simpler and rude in terms of the richness and hierarchy of colors.

\section{RESEARCH ON THE THEME OF "CHUBBY BABY" IN DONGFENGTAI NEW YEAR PAINTINGS}

\subsection{Baby Play Paintings in Dongfengtai New Year Paintings}

Among the Dongfengtai New Year paintings, a large number of people's yearning for a better life is pinned in the innocence and loveliness of children. For example, in the traditional paintings of Dongfengtai, there are "连年有余 (lian nian you yu, means to hope that the family will be wealthier and wealthier year by year), [冠带流传](liu chuan dai guan, means to hope that the people in this family can get hereditary officials, passed on from generation to generation), 八童过海 (ba tong guo hai, the eight children crossing the sea), and 五子戏鱼 (wu zi xi yu, the five children playing fish), as shown in "Figure 3".

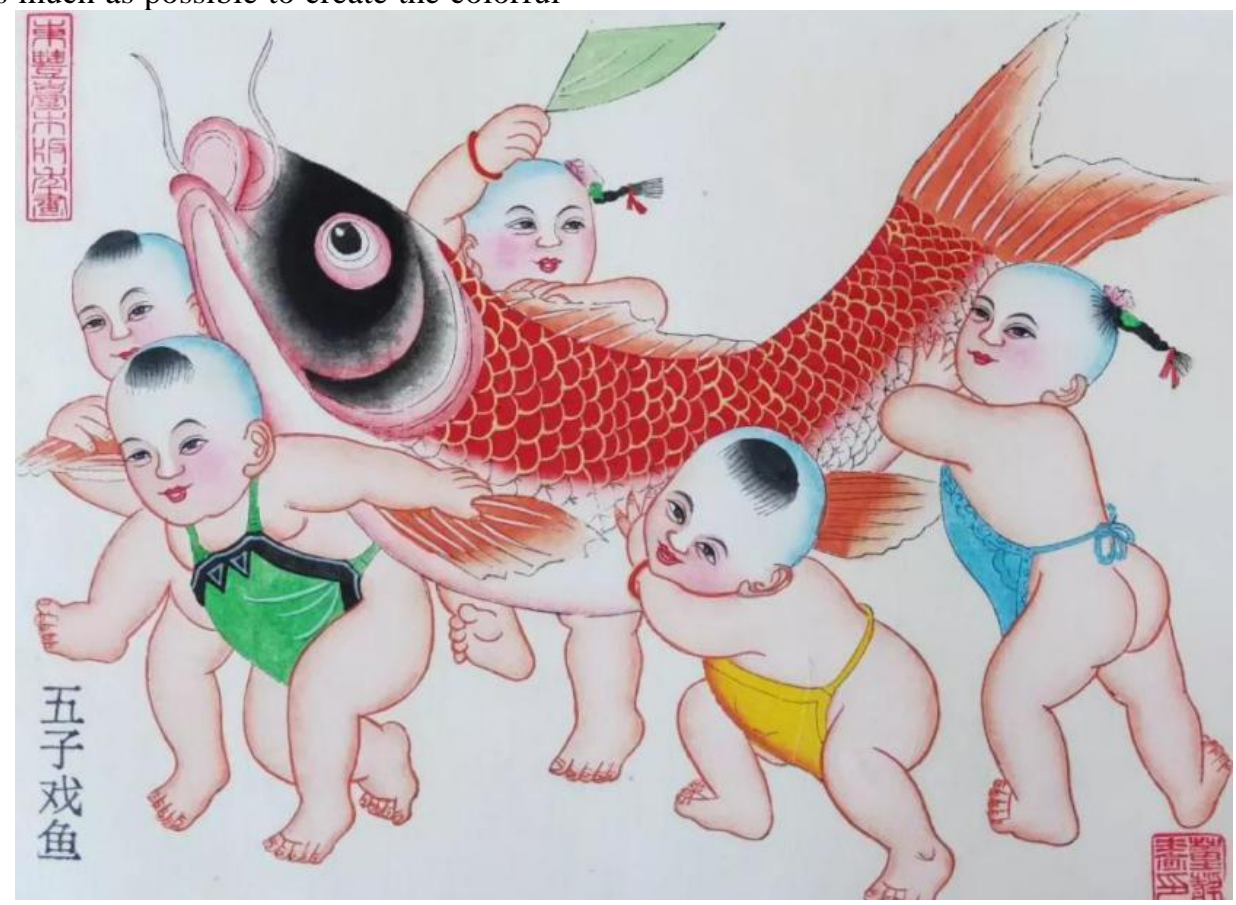

Figure 3 The five children playing fish.

At the same time, there are quite a lot of baby play paintings that have changed from worshipping and entertaining the gods to entertaining people. Children play games and play, etc. Among the many baby play paintings, there is no child with a sad face, only fresh and vivid, full of vitality. In the Dongfengtai New Year paintings, there are countless performances of this theme, such as walking on stilts and Taiping drums, as shown in "Figure 4". 


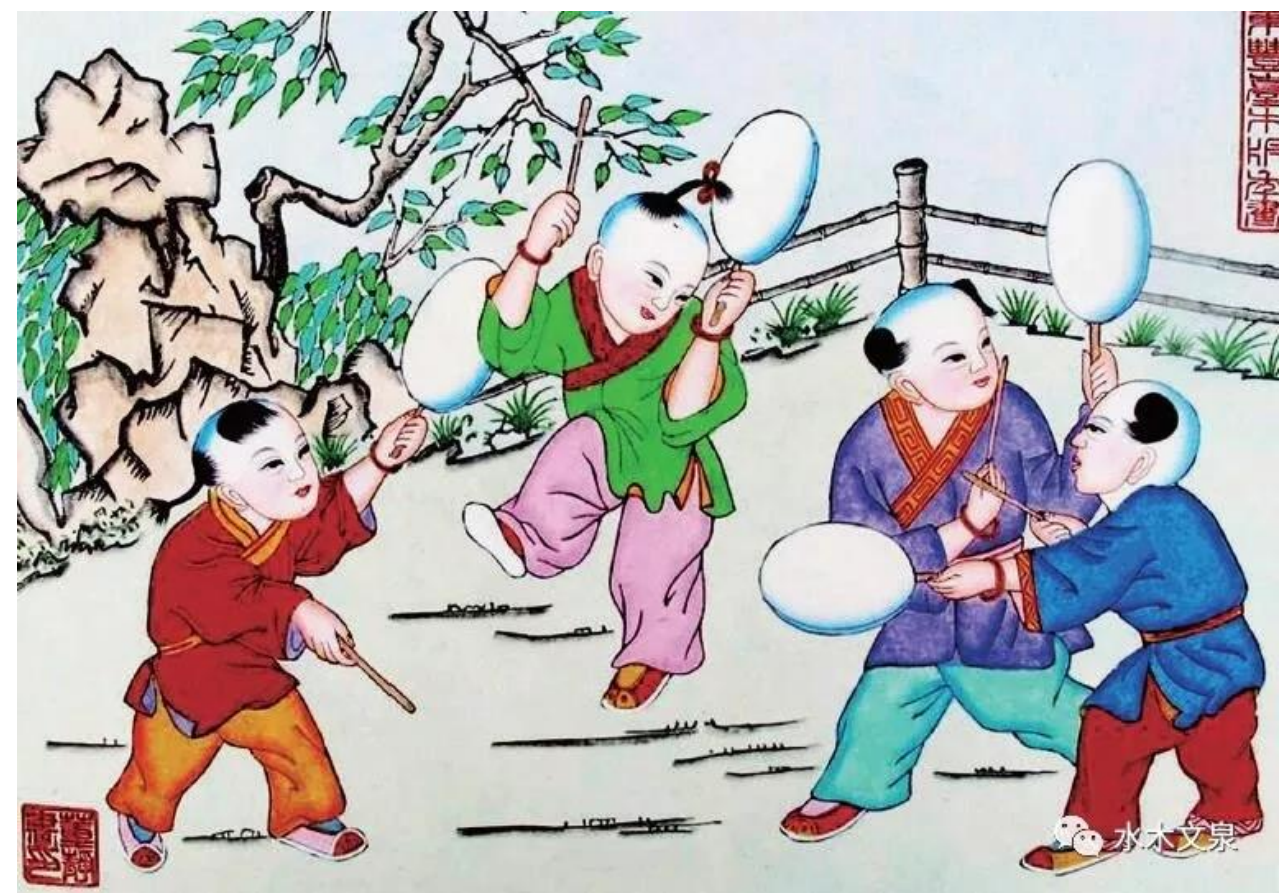

Figure 4 Taipin drum.

\subsection{Interpretation of the Image of the "Chubby Baby" in Dongfengtai New Year Paintings}

The image of "chubby baby" in Dongfengtai New Year paintings is unique. Compared with the image of "chubby baby" in Yangliuqing New Year paintings, the image of "chubby baby" in Dongfengtai New Year paintings is much rougher, not as cute as the babies in Yangliuqing New Year paintings, but more rustic and witty. Compared with Yangliuqing's model baby image, Dongfengtai New Year paintings are even more unrestrained. As shown in "Figure 5", in the Dongfengtai New Year paintings, the face of the baby is quite distinctive. Whether it is the similar Yangliuqing New Year paintings or the "chubby baby" in other New Year paintings, it has a special flavor.

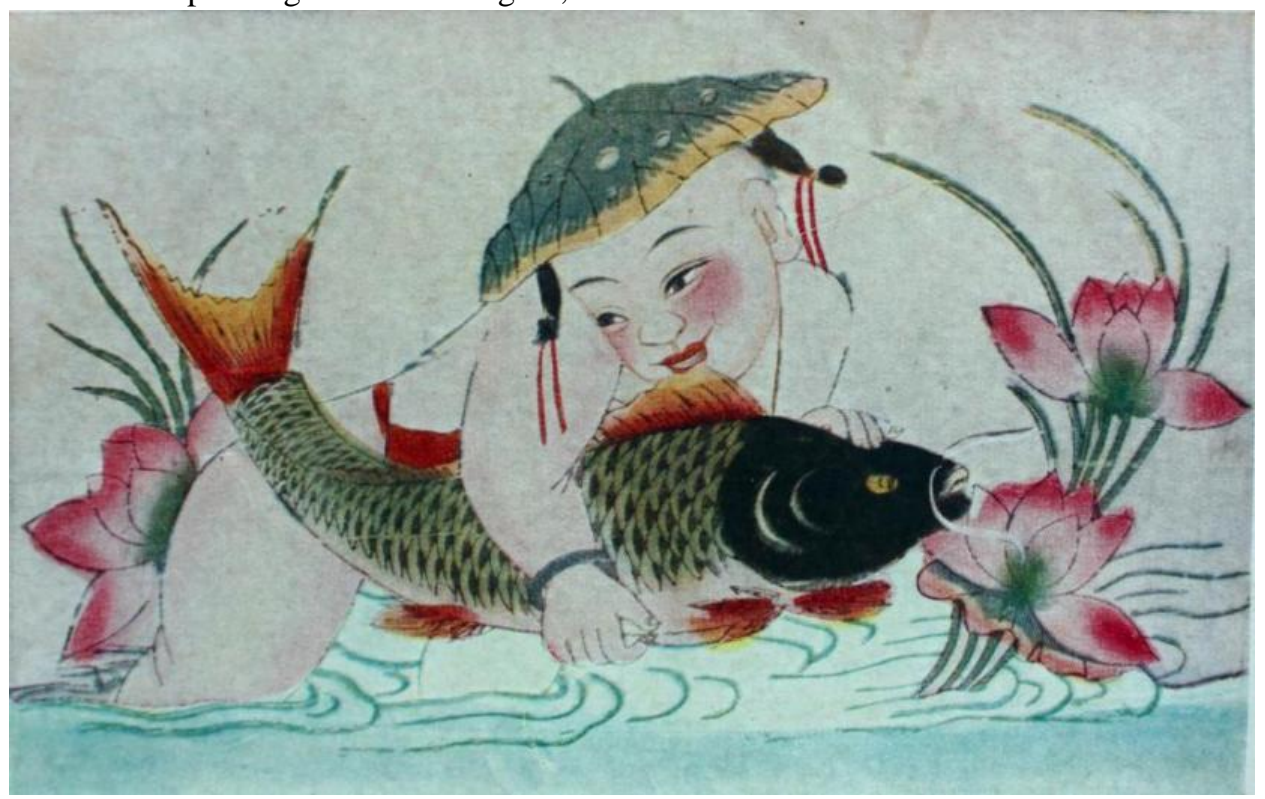

Figure 5 Lian nian you yu. 
The face in the Dongfengtai New Year paintings is an imitation of the Maitreya Buddha, and the face is a flat face, which is a derivative of the Maitreya Buddha; The ears of the baby are also the big ears of the Buddha statue, "大耳垂纶 (large and thick earlobe)". There is a saying in the folks: big ears symbolize blessing, so big ears can be said to be standard equipment; When observing carefully that there are teeth in the babies' mouths of Dongfengtai New Year paintings, this is also a feature of Dongfengtai New Year paintings, including the nearby Yangliuqing New Year paintings, which the chubby babies with teeth are not very common. This feature fits very well with the style of painting by folk painters, that is, they "paint what they have, and paint as well as it looks like". Although it is a bit simple, it is very appropriate to describe it; Eyes, the windows of the soul, in the Dongfengtai New Year paintings, the painters pay great attention to the depiction of the eyes, and in their hearts this finishing touch is the top priority, so the depiction of the eyes is generally very rich. The eyes of the babies in the Dongfengtai New Year paintings are "Yuanbao eye", and the eyes are in the shape of ingot turned upside down. The upper eye face is longer than the lower eye face, and the direction of the eye corner position line of the upper eye face is downward; the nose is a garlic nose. The garlic nose is in line with the cartoon image and looks very lively. There is also a saying in the folk that a big nose carries fortune. In Yangliuqing's paintings, the painters pay attention to "small nose, big eyes, no necks". ${ }^{5}[5]$ Compared with the "short arms, short legs, and no neck" in Yangliuqing New Year paintings, the baby's body in Dongfengtai New Year paintings is much more burly, and the body is more casual and slightly "silly".

In general, the chubby baby image in Dongfengtai New Year paintings was designed in accordance with the blessings of the common people to future generations, which is full of the meaning of beautiful yearning. In terms of image, the Dongfengtai New Year paintings try to follow the principle of "likeness", the objective depiction of the babies does not have too many abstract forms, and basically conforms to the objective laws of children. The overall style is also a simple way of expression, simple exaggeration and eye-catching, showing a different kind of simplicity. The arrangement of the entire "chubby baby" is a kind

5. Liu Jun, Study on Chinese Folk painting of the Lad Images [D]. Jiangsu: Soochow University, 2011. of beautiful sustenance in line with folk people's yearning for a better life and aesthetic concept.

\subsection{Auxiliary Foil Analysis of Auxiliary Patterns}

In the creation of dolls in Dongfengtai New Year paintings, there is not only a lively display of the image of "chubby baby", and the role of auxiliary patterns is also an indispensable ingredient. In general doll-type works, "chubby babies" are used as the visual theme of the New Year paintings, and supplementary patterns and extensions of meaning are added to complement the richness of the entire creation. On the one hand, the content of the work is enriched, which makes the painting lively and rich, and on the other hand, it enriches the connotation of the work for the sustenance of the meaning.

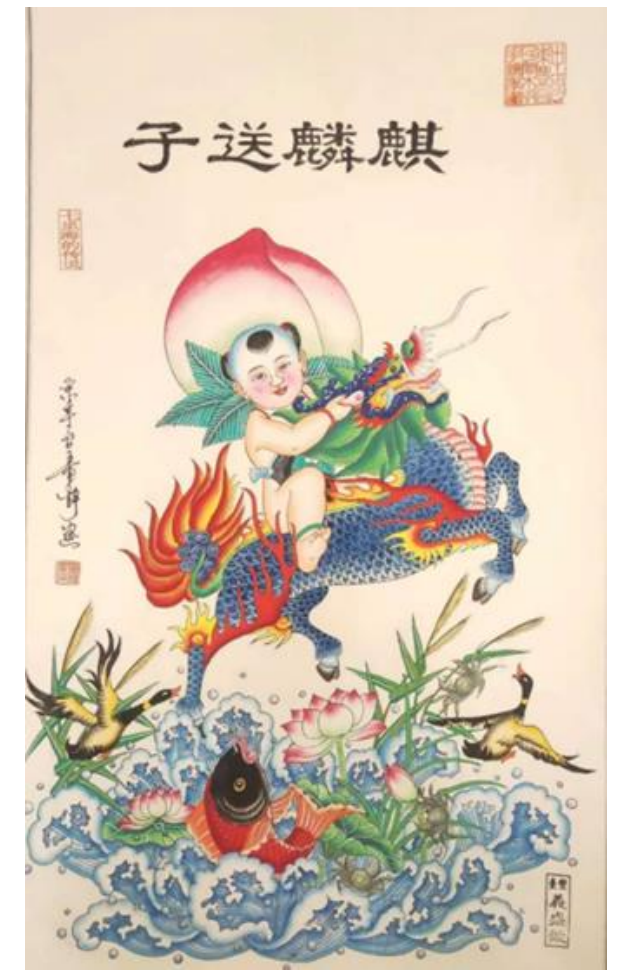

Figure 6 "Kirin Sending a Child" by Dong Jing.

Taking Mr. Dong Jing's "Kirin Sending a Child" as an example, as shown in "Figure 6". The visual center of the whole painting focuses on the bodies of "chubby baby" and Kylin as the main body of the work. The "chubby baby" image is vivid and lively. Sitting on the Kylin and pulling the Kylin's ears fully reflects the child's "naughty" image. In this sub-painting, the images of animals and plants are vividly represented, as if all the animals, plants, and rivers are vying to express their blessing. Animals 
include Kylins, carps, wild geese, and blue crabs, and plants include longevity peach, lotus, and reed grass. Each pattern has its own beautiful meaning and blessing.

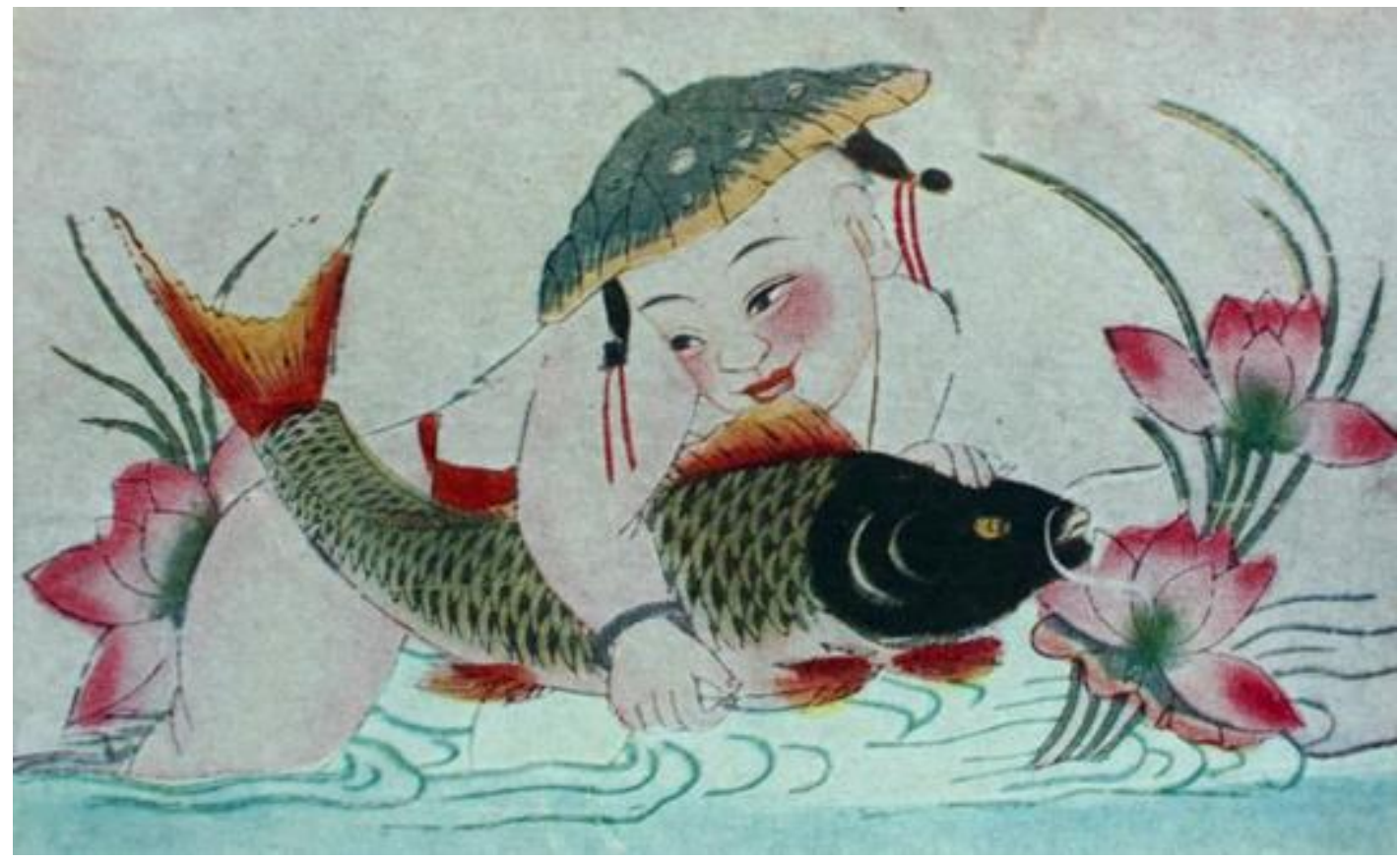

Figure 7 Lian nian you yu.

Fish represents "余 (yu)" in the folk, and there are countless folk idioms about fish. There is a big difference between the fish and the Yangliuqing New Year paintings in Dongfengtai New Year paintings. In Yangliuqing New Year paintings, fish images mostly use ornamental goldfish images, while in Dongfengtai New Year paintings, fish images use big carps in the river, as shown in "Figure 7". Wild goose also has a beautiful meaning in traditional culture, symbolizing benevolence. Therefore, the implication in this New Year painting is also very obvious. It is joy for a Kylin to send a son. Isn't it a fortune to send a filial son? Purple crab and reed belong to the local three treasures, "Ninghe has three treasures: whitebait, purple crab, and reed." The ancient city of Fengtai has the Huanxiang River passing through it, the Heilong River around the city outside, and the Jihe River in the southwest of the town, which is connected to the Huanxiang River. Although this place is not comparable to the Jiangnan water town, it is also criss-crossed. The three treasures of the Ninghe River are all products of this place. The appearances in the New Year paintings are also countless.

The patterns and decorations shown in Dongfengtai New Year paintings have their own image meanings, and local characteristics are added on the basis of traditional patterns to form a relatively complete decorative pattern system. The good meanings that the people have long enshrined in it fully reflect people's yearning for a better life and the continuous prayers of future generations.

\section{CONCLUSION}

Generally speaking, Dongfengtai New Year paintings do not seem to have a very high status in the current wood-panel New Year paintings. Compared with the Yangliuqing New paintings, the current volume is not worth mentioning, but it was once as famous as the Yangliuqing New Year paintings. The cultural form that has been preserved during the years of accumulation is also a treasure, with its own unique flavor and intriguing. At present, the author believes that the research atmosphere of Dongfengtai New Year paintings is not very optimistic, and it is very difficult to search for the collection and document inquiries. This aspect still needs to be added and strengthened. The content of Dongfengtai New Year paintings worthy of research and exploration is quite extensive. As Mr. Yang Yongzhi said, "The remnants of Dongfengtai woodblock New Year pictures are now the inklings, and rare. When we supply our generations for exploration and research, we should also try to protect and carry forward and keep pace with the times." 


\section{AUTHORS' CONTRIBUTIONS}

This article is independently completed by Jingyu Wang.

\section{REFERENCES}

[1] Xing Ye, The Newly Discovered Dongfengtai Yuelongzhuang New Year Paintings [J]. New Year Painting Research, 2011(0):128-130. (in Chinese)

[2] Feng Jicai, The Lost Dongfengtai New Year Paintings [N]. China Culture Daily, 2011-0920 (006). (in Chinese)

[3] A Ying, Collected Works of A Ying (Vol. 8) [M]. 2003. (in Chinese)

[4] Niu Jiazhi, Relying on Universities to Explore the New Development Path of BeijingTianjin-Hebei Woodcut New Year Paintings [J]. Inner Mongolia Science Technology \& Economy, 2018(20): 121-123. (in Chinese)

[5] Liu Jun, Study on Chinese Folk Painting of the Lad Images [D]. Jiangsu: Soochow University, 2011. (in Chinese) DOI:10.7666/d.y1990723. 